\title{
Erkrankungen an Lyme-Borreliose in den Jahren 1994 bis 1996
}

\author{
Erhebungen in den neuen Bundesländern
}

\section{Einführung}

Lyme-Borreliosen gehören zu den erst in jüngerer Vergangenheit näher charakterisierten Erkrankungen. Im Jahre 1975 wurde, ausgehend von einem gehäuften Auftreten einer Arthritis und weiterer unklarer Beschwerden, in der näheren Umgebung des Ortes Lyme (Connecticut, USA) diese Form der Borreliosen beschrieben. In den nachfolgenden Untersuchungen konnte ein Zusammenhang mit vorausgegangenen Zeckenbissen hergestellt werden. Die Isolierung des Erregers, einer bis dahin unbekannten Spirochäte, gelang Burgdorfer und Mitarbeitern im Jahre 1981, dem zu Ehren diese Spezies B. burgdorferi genannt wurde. Aufgrund genetischer Analysen wurde $B$. burgdorferi später in drei verschiedene Genospezies klassifiziert. Als Oberbegriff wurde $B$. burgdorferi "sensu lato « eingeführt; die drei Genospecies wurden $B$. burgdorferi sensu stricto, $B$. garinii und $B$. afzelii genannt.

$B$. burgdorferi wird durch Schildzecken der verschiedenen Spezies der Gattung Ixodes auf den Menschen übertragen. In Europa handelt es sich hierbei um Ixodes ricinus, den Gemeinen Holzbock. Dieser kommt in ganz Deutschland bis in $2000 \mathrm{~m}$ Höhe vor. Infizierte Zecken wurden bis zu einer Höhe von $1000 \mathrm{~m}$ gefunden.

Die Borrelien-Infektion erfolgt während des Blutsaugens über den Speichel der Zecke. In mehreren Untersuchungen wurde die Infektionsrate der Zecken in verschiedenen Regionen Deutschlands ermitrelt. Sie liegt zwischen 0 und ca. $40 \%[1,2]$. Als Erregerreservoir der Borrelien gelten vor allem Nager, Wild, Vögel, Igel und auch Haustiere [1].

Im Gegensatz zur Frühsommer-Meningoenzephalitis (FSME), einer wichtigen, ebenfalls durch Zecken übertragenen Viruserkrankung, ist die Borreliose nicht an begrenzte Naturherde gebunden. Borreliosen kommen in ganz Deutschland vor. Sie sind in Deutschland auch häufiger als die FSME.

Insbesondere die chronische oder nicht behandelte Infektion kann beim Men schen zu einem multisystemischen $\mathrm{Er}$ krankungsbild führen, wobei Haut, Gelenke, Herz und Nervensystem betrof fen sein können. Ein großer Teil der Borrelien-Infektionen verläuft jedoch inapparent.

Der klinische Verlauf der Borreliose kann in drei Stadien eingeteilt werden. Stadium I ist vor allem durch das Erythema chronicum migrans (ECM) charakterisiert. Für das Stadium II, das gegebenenfalls erst Wochen bis Monate nach dem Zeckenbiß auftritt, kann eine seröse Meningitis oder die sogenannte lymphozytäre Meningoradikulitis Bannwarth mit radikulären Schmerzen - mit oder ohne Lähmungserscheinungen - ebenso bestimmend sein wie eine Fazialisparese. Charakteristisch für das Stadium III sind die Lyme-Arthritis sowie die Acrodermatitis chronica atrophicans Herxheimer, die Monate bis Jahre nach einer Infektion auftreten können.
Die Letalität der Borreliose ist - in Abhängigkeit vom befallenen Organsystem - unterschiedlich, insgesamt aber sehr niedrig (< 1 Promill). Eine realistische Berechnung der Letalität ist schwierig, da selbst die Mehrzahl der symptomatischen Infektionen niche diagnostiziert wird.

\section{Register für Erkrankungen an Borreliose}

Für die Lyme-Borreliose besteht keine spezielle Meldepflicht gemäß BSeuchG. Allerdings sind Borreliose-Erkrankungen mit meningitischen Verläufen unter der Meldekategorie »andere bakterielle Meningitiden « in die Statistiken aufzunehmen. Diese liegen aber in der Regel im RKI ohne nähere Angaben zum Einzelfall vor. Einige Bundesländer machten allerdings von ihrem in $\$ 7$, Absatz 3 BSeuch $G$ vorgesehenen Recht auf Erlaß einer Verordnung Gebrauch und erließen eine Meldepflicht für Lyme-Bor reliose.

Die in einzelnen Ländern (Brandenburg, Mecklenburg-Vorpommern, Sachsen, Sachsen-Anhalt, Thüringen) erfaßten Borreliose-Erkrankungen werden dem RKI im Rahmen der wöchentlichen Meldungen übermittelt. Für den größten Teil dieser Erkrankungsfälle stehen detaillierte Angaben zum Einzelfall zur Verfügung, die ab 1994 in einem $\gg$ Register« zusammengestellt wurden.

$\mathrm{Da}$ zum gegenwärtigen Zeitpunkt die Daten aus den zurückliegenden drei Jahren für die Bundesländer Brandenburg,
Tabelle 1: Im Register erfaßte Erkrankungen an Borreliose

\begin{tabular}{lcccc}
\hline Bundesland/Jahr & 1994 & 1995 & 1996 & Summe \\
\hline Brandenburg & 32 & 42 & 86 & 160 \\
Mecklenburg- & 39 & 54 & 54 & 147 \\
Vorpommern & & & & \\
Sachsen & 15 & 30 & 175 & 220 \\
Sachsen-Anhalt & 54 & 75 & 82 & 211 \\
Thüringen & 19 & 33 & 28 & 80 \\
Summe & 159 & 234 & 425 & 818 \\
\hline
\end{tabular}

Tabelle 2: Gemeldete Erkrankungen an Borreliose

\begin{tabular}{lcccccc}
\hline Bundesland/Jahr & 1991 & 1992 & 1993 & 1994 & 1995 & 1996 \\
\hline Brandenburg & o. A. & o. A. & 36 & 34 & 43 & 86 \\
Mecklenburg- & 21 & 89 & 109 & 38 & 54 & 54 \\
Vorpommern & & & & & & \\
Sachsen & 67 & 79 & 41 & 16 & 31 & 175 \\
Sachsen-Arhalt & 46 & 96 & 54 & 59 & 74 & 82 \\
Thüringen & 12 & 30 & 41 & 18 & 43 & 28 \\
Summe & 146 & 294 & 281 & 165 & 245 & 425 \\
\hline
\end{tabular}


Mecklenburg-Vorpommern, Sachsen, Sachsen-Anhalt und Thüringen vorliegen, soll im folgenden eine erste Auswertung und Analyse der erhobenen Daten für den Zeitraum 1994 bis 1996 vorgenommen werden.

Anzumerken ist an dieser Stelle, daß es sich bei den im RKI gewonnenen Daten keinesfalls um eine vollständige Erfassung aller in dem betreffenden Zeitraum in dem entsprechenden Gebiet aufgetretenen Borreliosen handelt. Somit können die dargestellten Ergebnisse immer nur unter diesem Gesichtspunkt betrachtet werden. Beispiele anderer meldepflichtiger Erkrankungen zeigen, daß in den meisten Fällen nur ein Teil der tatsächlich aufgetretenen Erkrankungsfälle über den Meldeweg ermittelt werden kann.

\section{Ergebnisse}

Im Ergebnis der durchgeführten Erhebungen standen für die Jahre 1994 bis 1996 Angaben zu 818 Erkrankungsfällen an Borreliose in den neuen Bundesländern zur Verfügung. Die Verteilung der Erkrankungen auf die einzelnen Jahre sowie auf die fünf Bundesländer ist in Tabelle 1 dargestellt.

In den Jahren von 1994 bis 1996 kam es zu einem ständigen Anstieg der gemeldeten Zahlen. Bei der Betrachtung der Häufigkeit in den an den Erhebungen beteiligten Bundesländern wird deutlich, daß in diesem Zeitraum eine beträchtliche Steigerung der Zahl der gemeldeten Erkrankungen insbesondere in Sachsen erfolgte (von 15 Erkrankungen im Jahre 1994 auf 175 im Jahre 1996). Auch in Brandenburg und Sachsen-Anhalt war ein deutlicher Zuwachs in der Zahl der erfaßten Borreliose-Erkrankungen zu verzeichnen. In Thüringen und auch in Mecklenburg-Vorpommern hingegen wurden in diesem Zeitraum keine erheblichen Veränderungen in den Fallzahlen registriert.

Bezieht man auch den davorliegenden Zeitraum (1991 bis 1993) in die Betrachtungen ein, so zeigen sich auch dort erhebliche Schwankungen in der jährlich registrierten Zahl der Erkrankungen (Tab. 2). Die Unterschiede in der Meldehäufigkeit in den einzelnen Bundesländern werden deutlicher, wenn man für die einzelnen Jahre die Zahl der Erkrankungen pro 100000 Einwohner ermittelt (Abb. 1). ( $\mathrm{Zu}$ berücksichtigen sind hierbei allerdings die schon oben genannten Einschränkungen hinsicht-

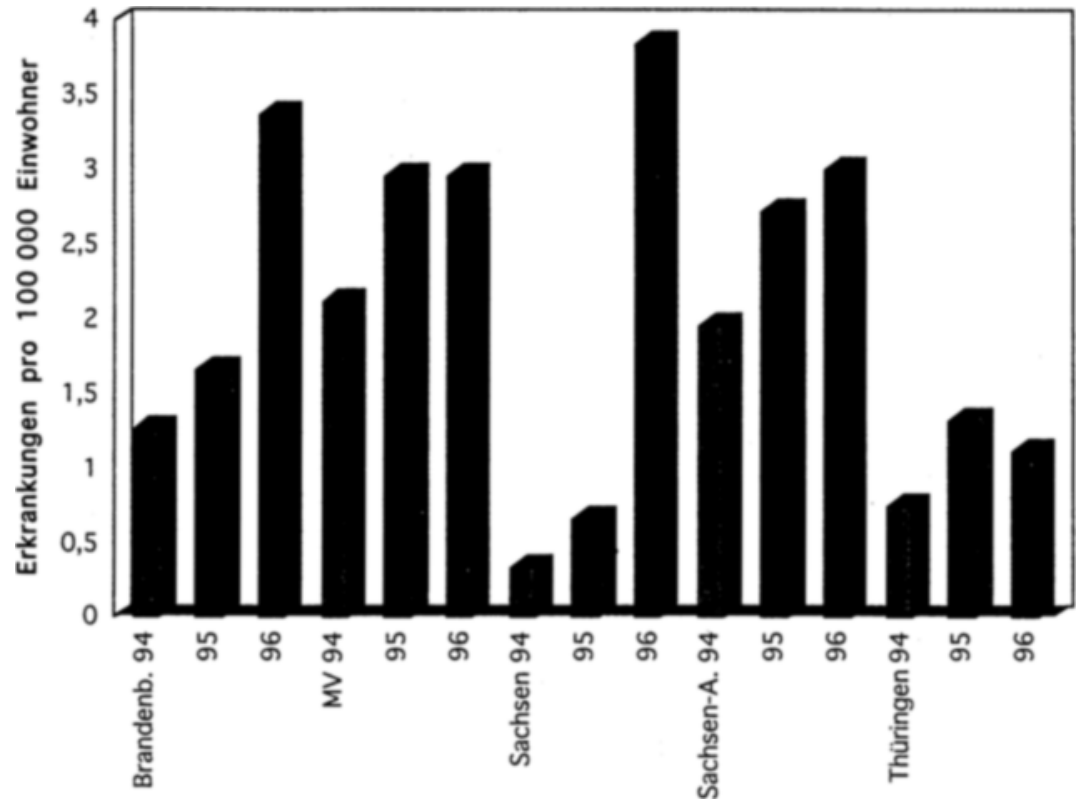

Abbildung 1: Borreliose-Erkrankungen 1994 bis 1996 in den Bundesländern.

lich des tatsächlichen Erfassungsgrades der Borreliosen.)

Die gemeldeten Borreliosen sind auf die verschiedenen Altersgruppen unterschiedlich verteilt (Abb. 2). Auffällig hierbei ist, daß die Gruppe der fünf- bis 14jährigen nach den vorliegenden Daten weitaus häufiger als andere Altersgruppen von einer akuten Borreliose betroffen ist. Ihr Anteil (ca. $25 \%$ ) an der Gesamtzahl der registrierten Erkrankungen übertrifft bei weitem den der anderen Altersgruppen. Jugendliche und

junge Erwachsene (15- bis 24jährige) erkranken nach diesen Erhebungen in nur geringerem Maße (Anteil ca. $7 \%$ ) an Borreliose. Bei den Erwachsenen ist bis zum 65. Lebensjahr eine Zunahme der Erkrankungshäufigkeit zu verzeichnen. Der Anteil von Borreliose-Erkrankungen bei Personen, die älter als 65 Jahre sind, nimmt jedoch in der vorliegenden Stichprobe wieder deutlich ab.

Im Rahmen dieser Erhebungen wurde auch ein Sterbefall infolge BorrelioseErkrankung registriert. Dieser trat im

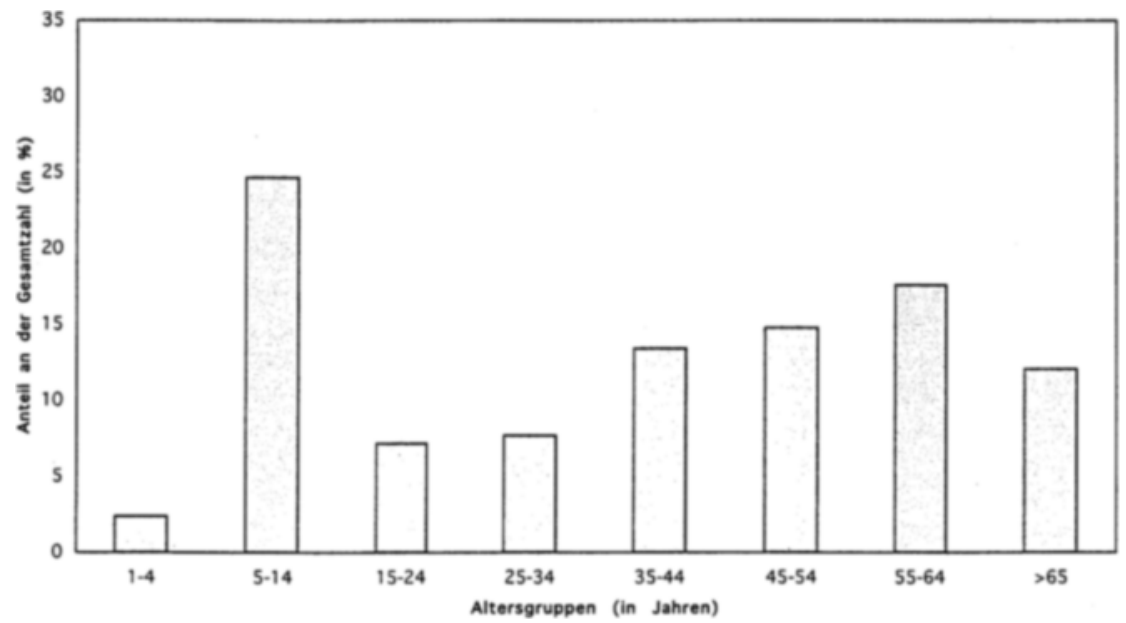

Abbildung 2: Borreliose-Erkrankungen 1994 bis 1996 - Verteilung nach Altersgruppen. 
Tabelle 3: Symptomatik der Borreliose-Erkrankungen 1994 bis 1996

\begin{tabular}{lcc}
\hline Symptomatik & $\begin{array}{c}\text { Zahl der Patienten } \\
\text { mit eingeschränkter } \\
\text { Symptomatik / nur } \\
\text { eines Organsystemes }\end{array}$ & Anteil in \% \\
\hline Allgemeine Infektsymptomatik & 117 & 14,3 \\
Symptome der Haut & 273 & 33,4 \\
ZNS-Symptomatik & 87 & 10,6 \\
Symptome des Herz-Kreislauf- & 8 & 1,0 \\
Systems & 71 & 8,7 \\
Symptome der Gelenke & 15 & 1,8 \\
ohne Angaben & 247 & 30,2 \\
Patienten mit Symptomen & & 100 \\
verschiedener Organsysteme & 818 & \\
Summe & & \\
\hline
\end{tabular}

Jahre 1995 in Sachsen auf. Es handelt sich dabei um einen 48jährigen Mann (Alkoholiker), bei dem die klinische Diagnose Neuroborreliose (mit peripherer Fazialisparese, Ataxie, Nystagmus und Lähmungserscheinungen) gestellt wurde. Eine serologische Bestätigung erfolgte. Das Ergebnis pathologisch-anatomischer Untersuchungen erbrachte deutliche Veränderungen des Kleinhirns. Ein Zeckenbiß war anamnestisch nicht ermittelt worden.

Männliche und weibliche Patienten sind von Borreliosen nach den im » Register" vorliegenden Ergebnissen in annähernd gleichem Maße betroffen: 389 männliche Patienten ( $48 \%$ ) stehen 429 weiblichen Patienten ( $52 \%$ ) gegenüber.

Bei insgesamt 401 Erkrankungen (entsprechend $49 \%$ ) konnte in der Anamnese ein Zeckenbiß ermittelt werden.

Die Diagnose »Borreliose« wurde für 801 von 818 Erkrankungen, das sind $98 \%$, durch Ergebnisse serologischer Testverfahren unterstützt.

Für die in das »Register « aufgenommenen Borreliosen wurden sehr unterschiedliche klinische Symptome angegeben. Diese reichen von einer eher unspezifischen Infektsymptomatik bis hin zu Meningitiden. 242 Patienten (entsprechend $30 \%$ ) mußten stationär behandelt werden. Die aufgetretenen Symptome wurden in fünf SymptomenKomplexen, differenziert nach den betroffenen Organsystemen, erfaßt.

Für ca. $30 \%$ der Erkrankungen wurden dabei Symptome mehrerer Organsysteme angegeben. Bei der Betrachtung ptomatik, wurde diese für 117 Patienten der erhobenen Symptome wird deutlich, daß Hautmanifestationen überwiegen (Tab. 3 und 4). An erster Stelle steht hierbei das Erythema chronicum migrans (ECM), als Frühsymptom einer Borreliose, das bei immerhin 276 Patienten (entsprechend $34 \%$ aller Patienten) vorhanden war. Eine Acrodermatitis chronica atrophicans, als Spätmanifestation der Borreliose, wurde dagegen nur bei elf Patienten berichtet.

Bei einem großen Teil der Erkrankten (insgesamt bei 262 Patienten) wurde eine "unspezifische Infektsymptomatik«, wie Fieber, Schwäche, Myalgie, Lymphknotenschwellungen, gefunden. Als alleinige Symptomatik, d.h. ohne Auftreten einer organspezifischen Sym. $(14,3 \%)$ angegeben.

Tabelle 4: Erfaßte Symptome bei den Borreliose-Erkrankungen 1994 bis 1996 (inklusive Doppelnennungen)

\begin{tabular}{lcc}
\hline Symptomatik & $\begin{array}{c}\text { Gesamtzahl der erfaßten } \\
\text { Symptome bei den Patienten }\end{array}$ & davon: \\
\hline Allgemeine Infektsymptomatik & 262 & ECM \\
Symptome der Haut & 412 & $\begin{array}{c}\text { Meningitis } \\
41\end{array}$ \\
ZNS-Symptomatik & 184 & Fazialisparese \\
& & 52 \\
Symptome des Herz-Kreislauf- & 18 & Arthritis \\
Systems & 151 & 40 \\
Symptome der Gelenke & & \\
ohne Angaben & 15 &
\end{tabular}

ZNS-Manifestationen wurden bei insgesamt 184 Patienten diagnostiziert. $\mathrm{Da}-$ yon waren 41 Personen an einer Meningitis erkrankt. Die Meningitiden betrafen zu einem großen Teil (66\%) die Altersgruppe der fünf- bis 14jährigen. Neuritiden und Paresen (insgesamt 52 Fälle von Fazialisparese) sowie die Diagnose "Neuroborreliose" (21 Erkrankungsfälle) wurden als postprimäre ZNS-Manifestationen angegeben.

Im Zusammenhang mit der Erfassung von bakteriellen Meningitiden wurden dem RKI im Zeirraum von 1994 bis 1996 insgesamt acht Fälle an Borrelien-Meningitiden aus Berlin gemeldet.

Symptome seitens der Gelenke traten bei 151 Patienten auf; eine nur auf die Gelenke begrenzte Symptomatik bei 71 $(8,7 \%)$. Neben der Arthritis der großen Gelenke (in 40 Fällen) wurden auch $\mathrm{Ge}$ lenkschwellungen oder "rheumatische Beschwerden « berichtet.

Befunde seitens des Herz-KreislaufSystemes wurden für 18 Patienten erhoben. Hierbei handelte es sich um Vorhofflimmern, Extrasystolie oder Myokarditis.

Die im Rahmen dieser Erhebungen registrierten Borreliosen weisen Unterschiede hinsichtlich ihrer jahreszeitlichen Erfassung auf (Abb. 3). So kam ein Großteil der Erkrankungen im III. bzw. IV. Quartal zur Meldung (40 bzw. $36 \%$ ). Auf die ersten beiden Quartale der Jahre 1994 bis 1996 hingegen entfielen insgesamt lediglich $24 \%$ der gemeldeten Erkrankungen.

Betrachtet man nur die Erkrankungsfälle, für die ein ECM, ein in der Regel 
ganz am Anfang des klinischen Verlaufes stehendes Symptom, angegeben wurde, so wird der jahreszeitliche $\mathrm{Zu}$ sammenhang noch deutlicher. Diese Erkrankungen kamen zu $86 \%$ im III. und IV. Quartal zur Meldung.

\section{Diskussion}

Im Zeitraum der betrachteten drei Jahre kam es zu einem deutlichen Anstieg der gemeldeten Erkrankungszahlen an Borreliose. Unterschiede in der Zahl der järlich erfaßten Borreliose-Erkrankungen waren allerdings auch schon im Zeitraum von 1991 bis 1993 beobachtet worden.

Die Schwankungen in der Zahl der gemeldeten Borreliosen können durch verschiedene Faktoren verursacht werden. Grundsätzlich kann die Zunahme der gemeldeten Erkrankungszahlen sowohl durch eine bessere Diagnostik als auch durch eine vermehrte Meldung bedingt sein.

Für Sachsen ist davon auszugehen, daß der deutliche Anstieg der gemeldeten Erkrankungszahlen (von 16 im Jahre 1994 auf 175 im Jahre 1996) im Zusammenhang mit der Einführung der Verordnung über die Erweiterung der Meldepflicht im November 1995 steht. Ebenso hatte die Einführung einer Meldepflicht in Brandenburg (Verordnung vom 7. 10. 1996) möglicherweise schon einen Einfluß auf die Zahl der gemeldeten Fälle an Lyme-Borreliose im Jahre 1996 (43 im Jahre 1995, 86 im Jahre 1996).

Nicht auszuschließen sind auch witte rungsbedingte Einflüsse auf die Zecken, die Uberträger der Borrelien, die zu einem verstärkten Auftreten von Borreliose-Erkrankungen in einzelnen Jahren geführt haben könnten. Unklar bleibt der Anteil von Spätmanifestationen bzw. von Durchseuchungstitern bei den erfaßten Borreliosen, die möglicherweise sogar ohne Beziehung zur Symptomatik erhoben worden sein könnten.

Insgesamt liegen für Deutschland nur wenig aussagekräftige Zahlen zum Vorkommen von Erkrankungen an LymeBorreliose vor. Schätzungen zufolge treten in Deutschland jährlich ca. 30000 Lyme-Borreliose-Fälle auf [3]. Studien für Niedersachsen führten zu der Annahme, daß pro Jahr ein Erkrankungsfall pro 1000 bis 2000 Einwohner (entsprechend 50 bis 100 Erkrankungen pro

60

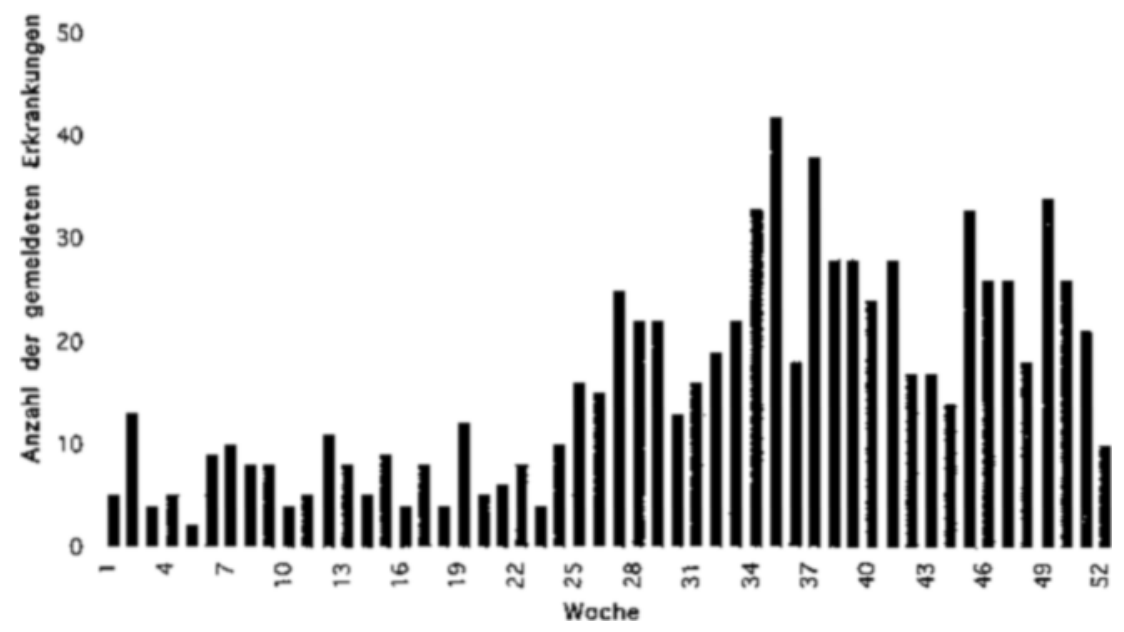

Abbildung 3: Borreliose-Erkrankungen 1994 bis 1996 - Verteilung auf die Meldewochen.

100000 Einwohner) in dieser Region auftritt. Hochrechnungen, die auf den Ergebnissen dieser Studie beruhen, lassen für Deutschland 40000 bis 80000 Erkrankungen an Borreliose pro Jahr möglich erscheinen [4].

Im Rahmen einer Studie, die 1995 unter Beteiligung von Kliniken und Diagnostiklaboratorien in Baden-Württemberg durchgeführt wurde, wurden insgesamt 97 Fälle von Borreliose registriert [5] Durch das Einbeziehen von 92 Kliniken (im Vergleich dazu 15 Diagnostiklaboratorien) ist allerdings ein Selektionseffekt (es werden zu einem großen Teil stationär Behandlungsbedürftige erfalft - insgesamt 65 Patienten) nicht auszuschließen. Gerade aber bei der Borreliose ist davon auszugehen, daß ein großer Teil der Patienten ambulant betreut wird [5].

Schwedische Autoren untersuchten für den Zeitraum eines Jahres die Verbreitung der Lyme-Borreliose in sieben Landkreisen Südschwedens. Aus den Untersuchungen ergab sich insgesamt eine Inzidenz von 69 Erkrankungsfällen pro 100000 Einwohner [6].

In anderen Regionen Europas, z. B. in Österreich, kann nach einer WHO-Publikation mit einer jährlichen Inzidenz von mehr als 200 Erkrankungsfällen pro 100000 Einwohner gerechnet werden [7].

In den USA wurden den CDC im Jahre 199511603 Fälle von Lyme-Borreliose gemeldet, was einer Inzidenz von 4,4
Erkrankungen pro 100000 Einwohner entspricht. In einzelnen Regionen wurden weitaus höhere Inzidenzraten (bis $\mathrm{zu} 45,6$ ) ermittelt [8].

Die mit Hilfe der dargestellten Registerdaten erhobene Altersverteilung der $\mathrm{Er}$ krankungen läßt verschiedene Möglichkeiten der Erklärung zu, gestattet jedoch keine eindeutigen Aussagen. Die Erhöhung der gemeldeten Erkrankungszahlen im Erwachsenenalter, die auch von anderen Autoren beschrieben wurde $[6,9]$, steht möglicherweise im Zusammenhang mit dem Ansteigen der Häufigkeit eines positiven Antikörpernachweises mit zunehmendem Alter. Den im Vergleich mit anderen Altersgruppen beobachteten größeren Anteil von Erkrankungen bei den fünf- bis 14jährigen bestätigen auch andere $\mathrm{Pu}$ blikationen. Dieser dürfte auch auf eine größere Expositionswahrscheinlichkeit in dieser Altersgruppe zurückzuführen sein. Allerdings läßt sich der niedrige Anteil von Personen, die älter als 65 Jahre alt sind, in dieser Stichprobe nicht erklären.

Eine Analyse der Erkrankungsfälle, für die ein Erythema chronicum migrans, eine für Borreliosen sehr typische Frühmanifestation, registriert wurde, erbrachte eine grundsätzlich gleiche Altersverteilung wie die für alle gemeldeten Borreliosen.

Für die im Rahmen dieser Erhebungen vorliegenden Erkrankungsfälle sind detaillierte Angaben zu den durchgeführten serologischen Bestimmungsmetho- 
den leider nicht bei allen Fällen vorhanden. Somit sind keine Angaben zu den verwendeten Methoden und damit zur Wertigkeit der erhaltenen Ergebnisse bekannt. Ebenso fehlen auch differenzierte Angaben zur ImmunglobulinKlasse der nachgewiesenen Antikörper (IgM oder IgG). Auf dieser Grundlage können für die hier beschriebenen Auswertungen keine Aussagen darüber gemacht werden, ob es sich in allen Fällen um eine frische Infektion gehandelt hat. Da die Diagnose "Borreliose « auch aufgrund des Nachweises schon länger vorhandener Antikörper gestellt worden sein kann, muß vermutet werden, daß es sich bei einem Teil der Erkrankungsfälle nicht um akute Borreliosen gehandelt haben könnte. Diese Vermutung wird unterstützt durch die für einen Teil der Erkrankungsfälle ange:gebene recht unspezifische Symptomatik (beispielsweise 117 Patienten mit »allgemeiner Infektsymptomarik o ohne weitere Symptome) bzw. die Angabe von Spätsymptomen. Möglicherweise sprechen hierfür auch die Unterschiede zwischen der Exposition, d. h. einem erinnerlichen Zeckenbiß (bei $49 \%$ der Erkrankten), und der Diagnostik einer Erkrankung an Borreliose.

Untersuchungen bei Patienten einer amerikanischen Klinik, bei denen zuvor Lyme-Borreliose diagnostiziert worden war, führten zu dem Ergebnis, daß nur $23 \%$ (180 von 788 Patienten) an einer aktiven Lyme-Borreliose erkrankt waren. Weitere $20 \%$ waren zu einem früheren Zeitpunkt an Lyme-Borreliose erkrankt. Der größte Teil der Patienten $(57 \%)$ war jedoch nicht an Borreliose erkrankt. Bei $45 \%$ von ihnen war in einem anderen Labor $\mathrm{zwar}$ ein positives Testergebnis erhalten worden; bei erneuter Untersuchung im Labor der Autoren erwiesen sich diese Patienten jedoch als seronegativ [10].

Problematisch ist zum gegenwärtigen Zeitpunkt die fehlende Standardisierung der Diagnostik. Die Bewertung positiver serologischer Befunde ist schwierig, besonders in Gebieten mit hoher Durchseuchung. Erforderlich ist eine entsprechend vorsichtige Interpretation positiver Befunde bei Patienten mit vieldeutigen klinischen Beschwerden. Andererseits kann auch der AntikörperNachweis, insbesondere bei Patienten mit noch kurzer Krankheitsdauer, nicht selten negativ ausfallen [11].

Nach Kaiser und Mitarbeiter läßt sich die Diagnose einer Borreliose nur mit ei- ner gewissen Wahrscheinlichkeit stellen. Sie schlagen deshalb vor, die Diagnose der Borreliose in Abhängigkeit vom Umfang der Informationen als möglich, wahrscheinlich oder sicher einzustufen [5].

Durchseuchungsstudien, durchgeführt in verschiedenen deutschen Bundesländern, erbrachten folgende Ergebnisse: Antikörper gegen Borrelia burgdorferi konnten bei 3-17\% der Untersuchten nachgewiesen werden (Studien in Bayern, Berlin und Nordrhein-Westfalen) Eine regional unterschiedlich hohe Prävalenz von Borrelien-Antikörpern bei klinisch Gesunden wird auch von Lücking 1996 [12] hervorgehoben, der für den Freiburger Raum ca. $20 \%$ angibr. Bei Beschäftigten mit hohem Risiko für Zeckenstiche (Forstarbeiter) wurden Antikörper bei 14-34 \% der getesteten Personen in Bayern und Hessen nachgewiesen [13]. Untersuchungen in Mecklenburg-Vorpommern erbrachten eine Antikörper-Prävalenz von $25 \%$ (indirekter Hämagglutinationstest) bei Wald- und Forstarbeitern und von $8 \%$ in der Normalbevölkerung [14]. Ähnliche Ergebnisse zeigten ebenfalls Untersuchungen, die bei Waldarbeitern des Bundeslandes Brandenburg durchgeführt wurden $[15,16]$. Hier wurden im IgG-Immunoblot positive Befunde bei immerhin $18 \%$ der untersuchten, in fünf verschiedenen Forstdistrikten beschäftigten Waldarbeiter gefunden. Im Vergleich hierzu wurden positive Befunde nur bei $5 \%$ der Kontroligruppe nachgewiesen.

Im Rahmen der im RKI durchgeführten Erhebungen liegen keine Angaben zum verursachenden Erreger vor. Unbekannt ist, ob in einigen Fällen auch eine Erreger-Spezies-Diagnostik durchgeführt wurde. Literaturberichten zufolge wird gegenwärtig versucht, eine Zuordnung einzelner Spezies zu bestimmten Erkrankungsformen bzw. Symptomen vorzunehmen. Bisher erhobene Befunde deuten daraufhin, daß bei europäischen Patienten $B$. afzelii überwiegend aus Hautbiopsien (80-90\%) und B. garinii aus Liquor cerebrospinalis (ca. $70 \%$ ) isoliert werden kann [1].

Bei der Betrachtung der klinischen Symptome wird deutlich, daß für die in das "Register" aufgenommenen Erkrankungsfälle an Borreliose, dem multisy stemischen Erkrankungsbild entsprechend, sehr unterschiedliche Sympromenkomplexe angegeben wurden. Ist für eine Vielzahl von Fällen nur eine eher unspezifische "allgemeine Infektsymptomatik « bekannt, so stehen dem ebenso Fälle von Meningitis, Arthritis oder Herz-Kreislauf-Beschwerden gegenüber. An erster Stelle in der Häufigkeit der angegebenen Symptome stehen jedoch Hautmanifestationen, die von einem »typischen« ECM, über Hautrötungen bis hin zur Acrodermatitis chronica atrophicans reichen.

Leider erweist sich die Auswertung der angegebenen Symptomatik als schwierig, da die für das Register zur Verfügung stehenden Angaben nicht standardisiert sind und einen möglicherweise längeren Krankheitsverlauf mit aufeinanderfolgenden verschiedenen Organmanifestationen nicht in adaquater und ausreichender Weise widerspiegeln können.

Für weitere Auswertungen wäre es wünschenswert, die Qualität der für das Register verwendeten Daten weiter zu verbessern. Das betrifft insbesondere die Aussagen zur serologischen Testung (Testmethoden, Ig-Klassen) sowie die Angaben zur Klinik. Notwendig wäre es, die Erkrankungen in ihrem Verlauf detaillierter zu erfassen.

\section{Anmerkungen zur Melde- pflicht nach BSeuchG}

Im Gegensatz zur Lyme-Borreliose, die nicht zu den meldepflichtigen Erkrankungen gehört, sind Erkrankungen an epidemischem Rückfallfieber, hervorgerufen durch Borrelia recurrentis sowie an endemischem Rückfallfieber (B. hermsii, B. turicatae, B. duttonii u. a.) nach $\$ 3$ BSeuch G meldepflichtig. Rückfallfieber hat in unseren Breiten, ebenso wie einige andere meldepflichtige Zoonosen auch - Pest, Fleckfieber, Rotz seit langem keine praktische Bedeutung mehr [17]. Jedoch wurden für die Jahre 1986 bis 1989 in der Bundesrepublik Deutschland insgesamt 27 Erkrankungen an Rückfallfieber ausgewiesen, die vermutlich als Lyme-Borreliose einzuordnen gewesen wären [18-20]. Da auch im Laufe der letzten Monate immer wieder vereinzelt Erkrankungsfälle an Rückfallfieber im Rahmen der wöchentlichen Meldungen weitergegeben wurden, die sich bei Nachfragen unsererseits in allen Fällen als Lyme-Borreliosen erwiesen, soll an dieser Stelle noch einmal darauf hingewiesen werden, daß Erkrankungen, verursacht durch Borrelia 
burgdorferi, der Borreliose und nicht dem Rückfallfieber zuzuordnen sind. Weiterhin läßt fehlendes Fieber in den genannten Fällen die Diagnose »Rückfallfieber a a priori äußerst unwahrscheinlich erscheinen. Ebenso kann die Erhebung der Reiseanamnese wichtigen Aufschluß über die Zuordnung der Erkrankungen geben, da Rückfallfieber in Deutschland nicht endemisch ist.

\section{Literatur:}

[1] Lyme-Borreliose. Erkennung und Verhütung. Merkblatt für Ärzte (Stand: 1996). Hrsg.: Bundesinstitut für gesundheitlichen Verbraucherschutz und Veterinärmedizin und Robert Koch-Institut, Bundesinstitut für Infektionskrankheiten und nicht übertragbare Krankheiten. Köln: Deutscher ÄrzreVerlag GmbH.

[2] Maiwald, M., Perney, T. N., Brückner, M. Krämer, C., Röhler, B., Beichel, E., und Hassler, D.: Untersuchungen zur natürlichen Epidemiologie der Lyme-Borreliose anläßlich des gehäuften Auftretens von Erkrankungen in einem Vorort einer nordbadischen Gemeinde. Gesundh.-Wcs. 57 (1995) 419-425.

[3] Süß, J. (Hrsg.): Durch Zecken übertragbare Erkrankungen: FSME und Lyme-Borreliose - Vorwort. Schriesheim: Weller, 1995.

[4] Horst, H. (Hrsg.): Einheimische Zeckenborreliose (Lyme-Krankheit) bei Mensch und Tier. Nürnberg: PERIMED-spitra, Med. Verl.-Ges., 1993.
[5] Kaiser, R., Kern, A., Fressle, R., Steinbrecher, A., Omran, H., Malzacher, V., Kügler, D. Kampa, D., und Batsford, S.: Zeckenvermittelte Erkrankungen in Baden-Württemberg Münch. med. Wschr. 138, 39 (1996) 647-652.

[6] Berglund, J., Eitrem, R., Ornstein, K., Lind berg, A., Ringner, A., Elmrud, H., Carlsson, M., Runehagen, A., Svanborg, C., and Norrby, R.: An Epidemiologic Study of Lyme disease in Southern Sweden. New Engl. J. of Med. 333, 20 (1995) 1319-1324.

[7] Anonym: Zoonoses control. Diagnosis and surveillance of Lyme borreliosis in humans. Weekly Epidemiological Record. 70, 43 (1995) 305-308.

[8] Anonym: Lyme Disease - United States, 1995. MMWR Vol. 45, No. 23 (1996) 481-484

[9] Hassler, D., Zöller, L., Haude, M., Hufnagel, H. D., und Sonntag, H. G.: Lyme-Borreliose in einem europäischen Endemiegebiet. Antikörperprävalenz und klinisches Spektrum. Disch. med. Wschr. 117, 20 (1992) 767-774.

[10] Steere, A. C., Taylor, E., McHugh, G., and Logigian, E. L.: The Overdiagnosis of Lyme Disease. JAMA 269, 14 (1993) 1812-1816.

[11] Wilske, B.: Diagnostik der Borrelia-burgdorferi-Infektion. Internist 36 (1995) 114-119.

[12] Lücking, C. H.: Diagnostische Crux? (Editorial). Münch. med. Wschr. 138, 39 (1996) 641-642.

13] Rath, P.-M.: Die Labordiagnostik der LymeBorreliose - Eine Übersicht. In: Durch Zecken übertragbare Erkrankungen: FSME und Lyme-Borreliose (Hrsg.: Süß, J.). Schriesheim: Weller, 1994.

[14] HülBe, C., Herrmann, H., Oheim, S., Schröder, L.-W., und von Stenglin, M.: Untersuchungen zur Verbreitung von Borrelia burg- dorferi in Mecklenburg-Vorpommern. Hyg. Med. 20 (1995) 345-350.

[15] Rath, P.-M., Ibershoff, B., Mohnhaupt, A., Albig, J., Eljaschewitsch, B., Jürgens, D., Horbach, I., und Fehrenbach, F. J.: Studie des Robert Koch-Instituts zur Seroprävalenz der Lyme-Borreliose bei Waldarbeiterinnen und Waldarbeitern in Brandenburg 1992/93. Bundesgesundhbl. 39 (1996) 414-415.

[16] Rath, P.-M., Ibershoff, B., Mohnhaupr, A., Albig, J.,Eljaschewitsch, B., Jürgens, D., Horbach, I., und Fehrenbach, F. J.: Seroprevalence of Lyme Borreliosis in Forestry Workers from Brandenburg, Germany. Eur. J. Clin. Microbiol. Infect. Dis. 15 (1996) 372-377.

[17] Weise, H.-J.: Zoonosen. Die aktuelle Bedeutung der Zoonosen im Rahmen meldepflichtiger Infekrionskrankheiten des Menschen. Bundesgesundhbl. 20, 24 (1977) 353-362.

[18] Pöhn, H.-P., und Großmann, R.: Infektionskrankheiten: Epidemiologische Situation 1986 und 1987 in der Bundesrepublik Deutschland. Bundesgesundhbl. 32, 1 (1989) 3-7.

[19] Pöhn, H.-P., und Großmann, R.: Infektionsepidemiologische Situation in der Bundesrepublik Deurschland 1988. Bundesgesundhbl. 33, 2 (1990) 43-46.

[20] Pöhn, H.-P., Zastrow, K.-D., und Großmann, R.: Infektionskrankheiten: Epidemiologische Situation 1989 in der Bundesrepublik Deutschland. Bundesgesundhbl. 34, 5 (1991) 199-202.

Dr. Gernot Rasch, Dr. Irene Schöneberg, Dr. Lothar Apitzsch, Robert Koch-Institut, Fachgebiet Meldepflichtige Krankheiten, Berlin

\title{
Die Verwendung von Unsicherheitsfaktoren in der quantitativen Risikoeinschätzung - neue Wege
}

\author{
Zusammenfassung der Ergebnisse eines BgVV-Workshops, \\ 5.-7. Mai 1997, Berlin-Marienfelde
}

\section{Die Verwendung von Unsicherheitsfaktoren}

Traditionellerweise werden Unsicher heitsfaktoren in der quantitativen Risikoeinschätzung verwendet, um sehr unterschiedlichen Arten von Unsicherheit Rechnung zu tragen. Nach Auffassung einiger Teilnehmer des Workshops ist die Verwendung solcher Faktoren seien sie nun fixe Faktoren im Sinne von Defaultwerten oder seien sie wissenschaftlich durch experimentelle Daten fundiert - trotz der weiten Verbreitung und der traditionellen Anwendung bei genauerer Untersuchung des der Unsicherheit zugrunde liegenden Sachverhaltes nicht durchgängig geeignet und in einigen Fällen auch theoretisch-wissenschaftlich nicht haltbar.

\subsection{Unsicherheit der experimentellen Daten}

Statistische Unsicherheit: Es ist ein bekanntes Problem, daß experimentelle Ergebnisse den wahren Wert nur un- vollkommen wiedergeben. Es ist daher wissenschaftliche Gepflogenheit, nicht nur ein Experiment auszuführen, sondern durch mehrfache Experimente ein Maß für den zufälligen Fehler und die Möglichkeit zu bekommen, das Zahlenintervall, in welchem der wahre Wert unter Angabe einer bestimmten statistischen Sicherheit liegt, angeben zu können. Unter der Annahme, daß kein systematischer Fehler das Ergebnis verzerrt, handelt es sich hier um ein übliches statistisches Problem, das nicht 